\title{
Delayed and highly specific antibody response to nonstructural protein 1 (NS1) revealed during natural human ZIKV infection by NS1-based capture ELISA
}

Xiujie Gao ${ }^{1}$, Yingfen Wen ${ }^{1}$, Jian Wang ${ }^{1}$, Wenxin Hong ${ }^{1}$, Chunlin Li $^{1}$, Lingzhai Zhao ${ }^{1}$, Chibiao Yin ${ }^{1}$, Xia Jin², Fuchun Zhang ${ }^{1 *+}$ and Lei $\mathrm{Yu}^{1 *+}$

\begin{abstract}
Background: Zika virus (ZIKV) had spread rapidly in the past few years in southern hemisphere where dengue virus (DENV) had caused epidemic problems for over half a century. The high degree of cross-reactivity of Envelope (E) protein specific antibody responses between ZIKV and DENV made it challenging to perform differential diagnosis between the two infections using standard ELISA method for E protein.

Methods: Using an IgG capture ELISA, we investigated the kinetics of nonstructural protein 1 (NS1) antibody response during natural ZIKV infection and the cross-reactivity to NS1 proteins using convalescent sera obtained from patients infected by either DENV or ZIKV.

Results: The analyses of the sequential serum samples from ZIKV infected individuals showed NS1 specific Abs appeared 2 weeks later than E specific Abs. Notably, human sera from ZIKV infected individuals did not contain cross-reactivity to NS1 proteins of any of the four DENV serotypes. Furthermore, four out of five NS1-specific monoclonal antibodies (mAbs) isolated from ZIKV infected individuals did not bind to DENV NS1 proteins. Only limited amount of cross-reactivity to ZIKV NS1 was displayed in 108 DENV1 immune sera at 1:100 dilution.
\end{abstract}

Conclusions: The high degree of NS1-specific Abs in both ZIKV and DENV infection revealed here suggest that NS1-based diagnostics would significantly improve the differential diagnosis between DENV and ZIKV infections.

Keywords: Zika virus, Dengue virus, Non-structural protein 1, Antibody response, Cross-reactivity

\section{Background}

With the rapid spread of Zika virus (ZIKV) in the Americas in 2015-2016, and its association with fetal skull malformations and neurologic disorders in adults, WHO declared ZIKV a global emergency [1, 2]. A total of 18 imported cases were reported during the year 2016 in China, with 12 cases in Guangdong, south China [3-6]. Historically, Guangdong had sporadic cases of dengue virus (DENV) infection for decades with the serotype 1 of DENV (DENV1) predominant in circulation

\footnotetext{
*Correspondence: gz8hzfc@126.com; leiyu11393@126.com

${ }^{\dagger}$ Fuchun Zhang and Lei Yu contributed equally to this work.

${ }^{1}$ Guangzhou Eighth People's Hospital, Guangzhou Medical University, 627

Dongfeng Rd. East, Guangzhou 510060, China
}

Full list of author information is available at the end of the article
[7-9]. A major DENV1 outbreak in 2014 in Guangdong had resulted in 13,800 hospitalizations with an estimated 300 cases of severe dengue and five reported deaths [9]. The preexisting DENV immune status in population combined with the risk of endemic spread of ZIKV infection, have raised the question of how to diagnosis each specific infection, and whether disease severity will be altered due to a potential antibody cross-reactivity because of the genetic and structural closeness between these two flaviviruses.

The flavivirus E protein is the main target of human antibody response. It contains three domains, EDI, EDII and EDIII [10]. The high cross-reactivity between ZIKV and DENV E-specific Abs was commonly known because of the similarity of their $\mathrm{E}$ proteins in sequence

(c) The Author(s). 2018 Open Access This article is distributed under the terms of the Creative Commons Attribution 4.0 International License (http://creativecommons.org/licenses/by/4.0/), which permits unrestricted use, distribution, and reproduction in any medium, provided you give appropriate credit to the original author(s) and the source, provide a link to the Creative Commons license, and indicate if changes were made. The Creative Commons Public Domain Dedication waiver (http://creativecommons.org/publicdomain/zero/1.0/) applies to the data made available in this article, unless otherwise stated. 
and structure [11-13], especially at an early time point during DENV and ZIKV infections [14, 15]. We have reported that the EDI/EDII binding Abs in sera from ZIKV infected individual peaked and waned earlier than EDIII binding Abs [15], consisting with the knowledge that ZIKV and DENV have a highly conserved fusion loop epitope (FLE) in EDII. The monoclonal antibodies (mAbs) isolated from the plasma cell or memory B cell appearing at early ZIKV infection also showed cross-reactivity with DENV and binding to the EDI/EDII [15].

The non-structural protein 1(NS1) of flavivirus can be released from infected cell to the blood or expressed on the cell surface. NS1 is also highly antigenic and contributes to the human antibody response repertoire against the virus $[16,17]$. Recently, the NS1-based serological tests have been developed for distinguishing ZIKV from DENV infection [18-21]. But little is known about the NS1-specific Ab response during the course of the ZIKV infection with respect to its specificity, magnitude, and kinetics which are of great relevance for diagnostics [22, 23]. Here we established an IgG capture ELISA method using a recombinant full-length NS1 expressed in mammalian $293 \mathrm{~T}$ cells and examined the changes of NS1-specific Ab response and its cross-reactivity with four DENV serotypes in sequential plasma samples from the two Chinese travelers returning from South America where they contracted ZIKV infection. Furthermore, we isolated five NS1 monoclonal antibodies (mAbs) from these two ZIKV-infected individuals and examined the specificity of these mAbs. Finally, we investigated the binding to ZIKV NS1 in sera from a population of DENV1-infected individuals. Our results showed that a very limited cross-reactivity of human NS1 antibody response existed between ZIKV and DENV. The kinetics and specificity of NS1 Ab revealed here had important implications for NS1-based diagnostics and vaccine development.

\section{Methods}

\section{Patients and blood samples}

Sequential blood samples were collected from two Chinese travelers returning from South American with ZIKV infection [5, 15]. Both of them had mild clinical symptoms. Sampling time covered acute phase and early convalescent ( $\leq 100$ days after symptom onset) in both individuals, with one of them being followed up to over 1 year. ZIKV infection was diagnosed as virus RNA positive by the Chinese Center for Disease Control and Prevention. The isolated plasma or PBMC was preserved at $-80{ }^{\circ} \mathrm{C}$ or liquid nitrogen tank as appropriate. Additionally, a total of 108 sera were collected on the day 6 to 260 after symptom onset between Nov 2014 and Mar 2015, from patients with RT-PCR-confirmed DENV1 infection and hospitalized during the 2014 dengue epidemic in Guangzhou The primary DENV1 infection was diagnosed as being only anti-dengue IgM positive, whereas secondary infection being positive for both anti-dengue IgM and IgG or only IgG positive using acute-phase serum samples from patients [9].

Recombinant ZIKV and DENV NS1 proteins and Western blot analysis.

The full-length NS1 coding sequences of ZIKV (KY888678), DENV1 (KY911976), DENV2 (KY911978), DENV3 (KY911981) and DENV4 (KY911982) (Additional file 1) were amplified from clinical serum samples or virus stains from infected Chinese patients by RT-PCR and cloned into the pCDNA3.1 expression vector (Invitrogen). Sequence confirmed vector was then transfected into $293 \mathrm{~T}$ cells and the supernatant was collected on day 4-5. The expression of ZIKV or DENV NS1 protein with the D7 tag on C-terminal in the supernatant was analyzed by Western blot using antibody D7324(Cliniqa) [24, 25]. Recombinant envelope glycoprotein of ZIKV(KU820898), DENV1(KJ438296), DENV2(JX470186), DENV3(KF824903), and DENV4 (JQ822247) were produced according to the method described above with a D7-tag at the C-terminus.

\section{Isolation of ZIKV NS1 mAbs from memory B cells}

ZIKV NS1 monoclonal antibodies were isolated from the memory B cell culture method in vitro [15]. Briefly thawed PBMCs derived from the two ZIKV-infected individuals (on day188 in patient1 and day66 in patient 2) were stained with a panel of fluorescence-labeled Abs (IgD-FITC, CD19-ECD, CD27-PC7, CD38-APCA750, IgM-PB, and CD45-KO Beckman Coulter). Memory B cells gated as IgD IgM - CD27 + CD38 low were sorted into 96-well culture plates ( 25 to 50 cells/well) and cultured with supplements as described previously [15]. After 10 days, the culture supernatants were screened for the presence of ZIKV NS1 binding mAbs using capture ELISA described in next paragraph. VH and VL sequences were obtained from positive B-cell cultures by RT-PCR as described [26] and analyzed using the IMGT/V-Quest program.

\section{Capture ELISA analysis}

The capture ELISA was established as previously reported [27]. The anti-D7 antibody (D7324, Cliniqa) was pre-coated on 96-well ELISA plates. The next day, after washing the plates, culture supernatants containing the NS1 or E protein from ZIKV and DENV with the D7 tag on C-terminal were added to establish capture ELISA. The binding of serum samples or mAbs to NS1 was measured. The binding activity was detected by anti-human IgG labeled with horseradish peroxidase(HRP) and 3,3',5,5'-Tetramethylbenzidine(TMB) substrate. Negative controls were measured by adding supernatant from the $293 \mathrm{~T}$ cell culture without transfection. Each sample was 
tested in duplicates. Absorbance value was corrected by using the mean value of negative controls.

\section{Statistics analysis}

In ELISA analysis of NS1 binding, half-maximal effective concentrations (EC50) were calculated using the dose-response-stimulation model in GraphPad Prism (GraphPad Software Inc.). Significance analyzed by Tukey's multiple comparisons test following two-way ANOVA.

\section{Results}

\section{Expression of recombinant NS1 protein and} establishment of capture ELISA

To investigate the human antibody response to ZIKV NS1 protein, we first expressed the full length NS1 proteins of ZIKV and DENV1-4 in mammalian $293 \mathrm{~T}$ cells (Fig. 1a and Additional file 1) and collected the culture supernatants containing NS1 proteins, which were shown to be about $55 \mathrm{KD}$ as detected by the D7 tag antibody in western blot (Fig. 1b). These NS1 proteins were used to set up capture ELISA with D7 Ab pre-coated plates.

\section{Dynamics and cross-reactivity of NS1 antibody response during natural ZIKV infection}

To examine the dynamic changes of human NS1 antibody response at polyclonal level during the course of ZIKV infection, we measured the serum binding Abs in sequential samples from two ZIKV infected patients. Eight sequential plasma samples were obtained from Pt1 on day 4, 7, 15, $32,65,106,188$, and 322, and three were obtained from Pt2 on day 6,12 , and 66 after the onset of symptom. The binding of all these serum samples to DENV1-4 NS1 was also tested at a dilution of 1:100. Comparing to E specific $\mathrm{Ab}$ response reported previously in serum samples from the same patient [15] (Fig. 2a), the NS1 specific antibodies appeared later, became detectable on day 15 , also peaked later on day 106 and declined markedly during disease (Fig. 2b). Similar trends of E and NS1 specific $\mathrm{Ab}$ responses were observed in $\mathrm{Pt} 2$ with peaks on day 12 and day 66, respectively (Fig. 2c and d). In contrast to high cross-reactivity of serum antibodies to DENV1-4 E proteins, there were no detectable binding of serum antibodies to DENV1-4 NS1 from both patients at all time points at a serum dilution of 1:100(Fig. $2 b$ and $d$ ).

\section{Binding and specificity of NS1 mAbs derived from memory B cells of ZIKV infected patients}

To further dissect the polyclonal anti-NS1 responses, we isolated five NS1 mAbs from these two ZIKV infected patients by memory B cell culture method and characterized their CDR3 regions and pattern of reactivity (Table 1). Most of mAbs (4/5) showed strong binding to ZIKV NS1 with EC50 values ranging from 9.8 to $277.4 \mathrm{ng} / \mathrm{ml}$, and no reactivity to DENV 1-4 NS1 proteins (Fig. 3, Table 1). Only one mAb (1/5), ZKns4F10, cross-reacted with DENV 2 and DENV 4 NS1 with EC50 of $116.1 \mathrm{ng} / \mathrm{ml}$ and $351.4 \mathrm{ng} / \mathrm{ml}$, respectively; 10-30 fold less than that to ZIKV NS1 (10.9 ng/ml) (Fig. 3, Table 1), which can be explained by the partial sequence and structure similarity shared between ZIKV and DENV (Additional file 1).

\section{Global analysis of NS1 cross-reactivity in DENV1 infected individuals}

To investigate whether the lack of significant cross-reactivity between anti-ZIKV NS1 antibodies and anti-DENV NS1 antibodies is unique to a few selected patients, we performed NS1 serum binding assay on a total of 108 convalescent sera from DENV1 infection

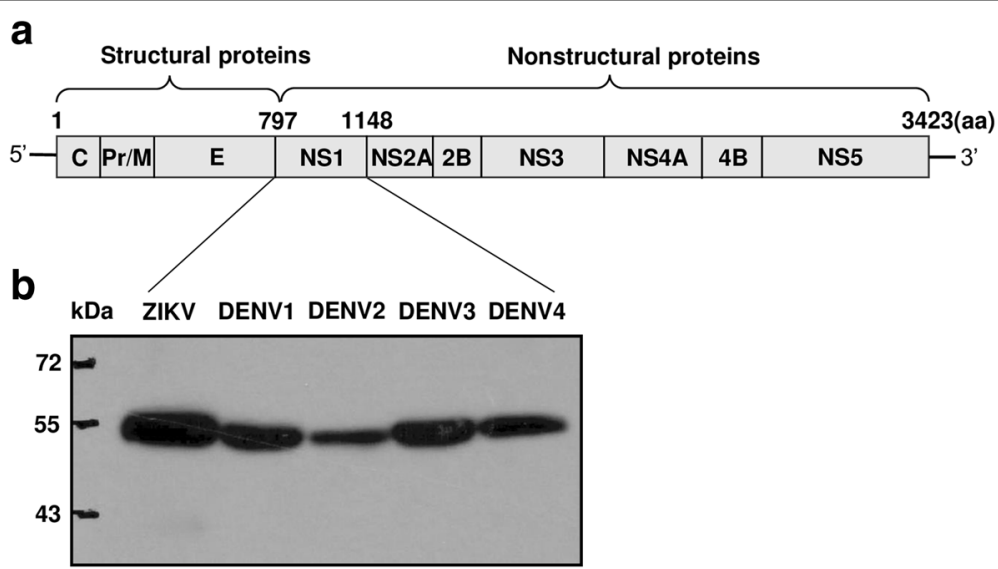

Fig. 1 The full-length NS1 proteins of ZIKV and DENV1-4 expressed in mammalian 293 T cells. a The structural and non-structural proteins of flavivirus. The schematic diagram showed the length of non-structure protein NS1 was about 352 aa. b Western blot analysis. ZIKV or DENV NS1 protein with the D7 tag on C-terminal was shown to be about 55KD as detected by the D7 antibody 


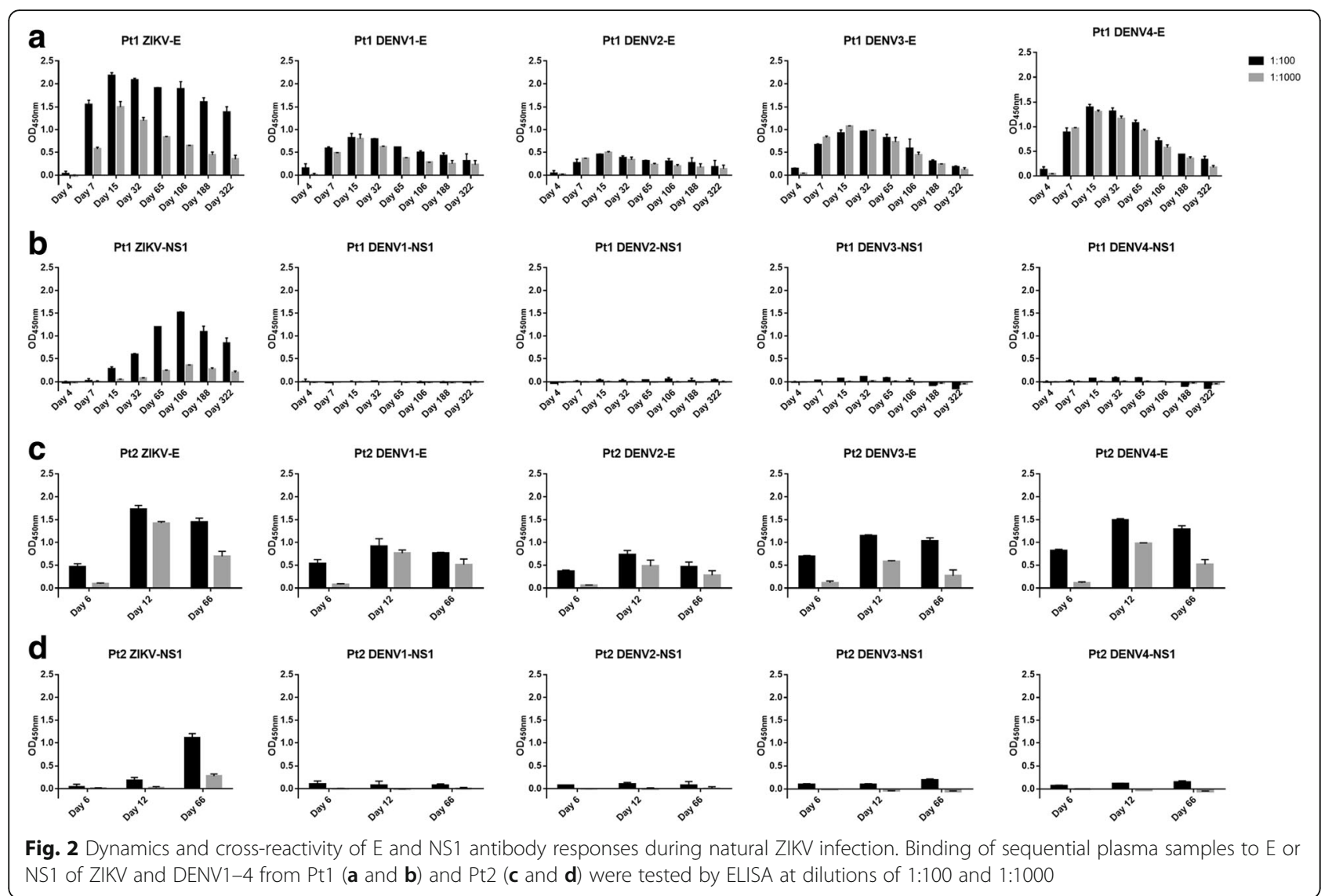

including primary infection $(n=35)$, secondary infec$\operatorname{tion}(n=20)$ and undetermined cases $(n=53)$ (Fig. 4). Lack of cross-reactivity to ZIKV was observed in all DENV1 infected individuals except for two cases which also exhibited significant cross-reactivity to DENV2, DENV3, and DENV4.

\section{Discussion}

Like in DENV infection, both E and NS1 proteins are targeted by the human immune system in ZIKV infection $[13,28]$. The extensive cross-reactivity between antibodies to ZIKV E protein and DENV E proteins of four different serotypes made it hard to differentiate between these two infections using the existing serology diagnostic tools. Several new NS1-based IgM/IgG ELISAs were reported for differential diagnosis of ZIKV and DENV infections $[18,21]$. Therefore, the understanding of NS1-specific Ab response during natural human ZIKV infection is urgent.

Recently, the changes of ZIKV-NS1 specific IgG levels during disease was described using the commercial ELISA kits [20, 29, 30]. In contrast, we reported for the first time the detailed kinetics of both E and NS1 antibody responses during nature ZIKV infection using sequential serum samples from the early phase, convalescence phase, and up to 1 year. Compared to anti-E responses, the NS1 specific Ab responses appeared later and peaked later. Both antibody responses,

Table 1 Specificity and genetic characteristics of mAbs isolated from two ZIKV infected individuals

\begin{tabular}{|c|c|c|c|c|c|c|c|c|c|c|c|c|c|}
\hline \multirow[t]{2}{*}{$\mathrm{mAb} I \mathrm{D}$} & \multirow[t]{2}{*}{ Origin } & \multirow[t]{2}{*}{ Day } & \multicolumn{5}{|c|}{ Binding to NS1 (EC $\left.C_{50}\right)(\mathrm{ng} / \mathrm{mL})$} & \multicolumn{3}{|l|}{ Heavy chain } & \multicolumn{3}{|c|}{ Light chain } \\
\hline & & & $\mathrm{ZIKV}$ & DENV1 & DENV2 & DENV3 & DENV4 & $\checkmark$ family & $\begin{array}{l}\text { CDR3 } \\
\text { sequence }\end{array}$ & $\begin{array}{l}\text { V gene } \\
\text { identity (\%) }\end{array}$ & $\checkmark$ family & CDR3 sequence & $\begin{array}{l}\text { V gene } \\
\text { identity (\%) }\end{array}$ \\
\hline ZKns3G2 & Pt1 & 188 & 34.5 & N/A & N/A & $\mathrm{N} / \mathrm{A}$ & $\mathrm{N} / \mathrm{A}$ & IGHV3-53 & ARERGWLDY & 93.68 & IGKV1-39 & QQTYTIPRT & 92.83 \\
\hline ZKns4F10 & Pt1 & 188 & 10.9 & N/A & 116.1 & N/A & 351.4 & IGHV4-31 & ARAIDNFYDNSI & 96.56 & IGKV1-39 & QQSYSPPYT & 94.98 \\
\hline ZKns2E11 & Pt2 & 66 & 9.8 & N/A & N/A & N/A & N/A & IGHV3-30-3 & ARVFNGYEGDY & 95.83 & IGLV3-10 & YSTDSSGNLYV & 97.49 \\
\hline ZKns14G5 & Pt2 & 66 & 277.4 & N/A & N/A & N/A & N/A & IGHV5-51 & ARSNVDGSTDY & 98.61 & IGLV3-25 & QSADSSDTYVPYV & 98.92 \\
\hline ZKns4B8 & Pt2 & 66 & 39.2 & N/A & N/A & N/A & N/A & IGHV3-53 & ASLGSGSAFGY & 96.84 & IGKV1-12 & QQANSFPFT & 98.21 \\
\hline
\end{tabular}



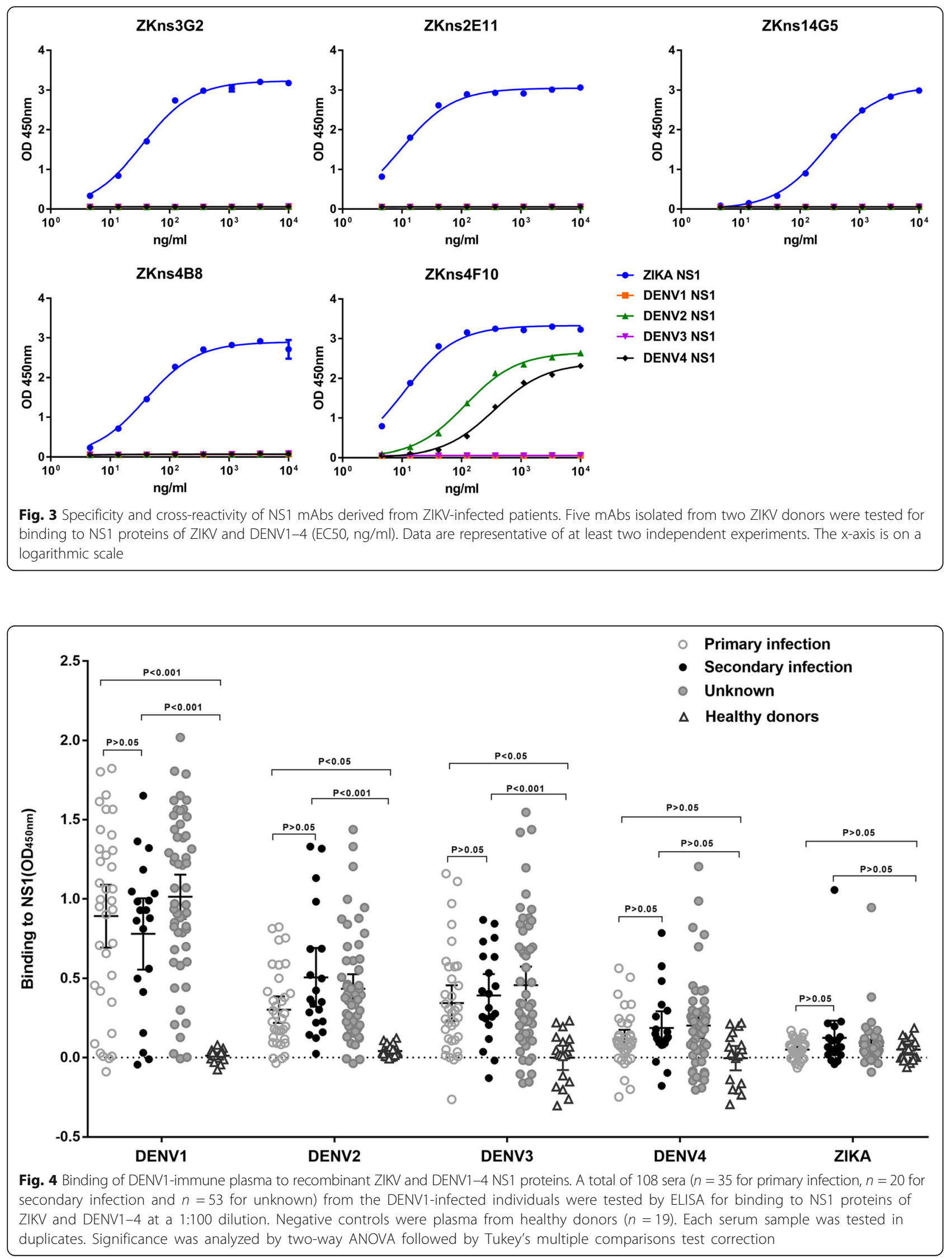
however, waned over time. The trend of NS1 antibody response in our study was consistent with the latest report in which the IgG positivity peaked between day 27 and day 61 [20]. Notably, there is a marked lack of cross-reactivity in the longitude sera samples from these two ZIKV-infected individuals to DENV NS1 proteins of all serotypes, indicating they did not have a prior DENV infection history [12].

In recent publication, most of $\mathrm{mAbs}(24 / 30)$ isolated from ZIKV-infected donors without previous exposure to DENV were ZIKV-specific, whereas mAbs(5/11) isolated from DENV-immune ZIKV-infected donors were cross-reactive [12]. In our study mAbs isolated from the two ZIKV infected patients showed high specificity. Among the five mAbs, four NS1 mAbs displayed ZIKV-specific with the lowest EC50 to $9.8 \mathrm{ng} / \mathrm{ml}$, which can be used to develop serological assays discriminating Zika virus from Dengue infection [19].

The cross-reactivity of DENV-immune sera to ZIKV-NS1 is a concern for serological test in ZIKV and DENV co-circulating areas. It was reported that 36-40\% plasma samples from ZIKV-naïve DENV-immune donors including secondary DENV infections displayed cross-reactivity with ZIKV, WNV, and YFV NS1 using a direct ELISA format [19]. We observed a limited serum cross-binding to ZIKV NS1 in a population of DENV1 infected individuals, even in those with secondary DENV1 infection, only $5 \%(1 / 20)$ showed binding to ZIKV NS1 by the established capture ELISA method. Sera from patients infected by other DENV serotypes are under investigation for cross-reactivity to ZIKV NS1 protein. Taken together, because of the high level of cross-reactivity between ZIKV and DENV E-reactive Abs, diagnostic strategies based on NS1 Abs are a valuable tool for diagnosing ZIKV infection where DENVs co-circulate [19, 20,31, 32].

\section{Conclusions}

By analyzing sera obtained from patients with ZIKV or DENV1 infection, we have discovered anti-NS1 antibodies being high specific at polyclonal and monoclonal levels, this is distinct from the high cross-reactive antibody responses to ZIKV and DENV E proteins. This observation supports the development of NS1 based diagnostic tools for areas where DENV and ZIKV co-circulate.

\section{Additional file}

Additional file 1: Sequence alignment of NS1 proteins of ZIKV and DENV1-4. Overall amino acid identity of each of the four DENV serotypes to ZIKV was shown on the top. Conservative regions were highlighted in color. The amino acid sequences encoding $\beta$-roll domain (1-30aa), wing domain (31-180aa), and $\beta$-ladder domain (181-352aa) were shown. (TIFF $872 \mathrm{~kb}$ )

\section{Abbreviations}

Abs: Antibodies; CDR3: Complementarity-determining region 3;

DENV: Dengue virus; DENV1: Dengue virus serotype 1; DENV2: Dengue virus serotype 2; DENV3: Dengue virus serotype 3; DENV4: Dengue virus serotype 4; E: Envelope protein; EC50: Concentration for $50 \%$ of maximal effect; ELISA: Enzyme-linked immunosorbent assay; FLE: Fusion loop epitope; IgG: Immunoglobulin G; IgM: Immunoglobulin M; mAbs: Monoclonal antibodies; NS1: Non-structural protein 1; WHO: World Health Organization; ZIKV: Zika virus

\section{Acknowledgments}

We thank Dr. Yongjun Guan for the consulting in the capture ELISA and the isolation of antibody.

\section{Funding}

This project was supported by funds from 2017 Guangzhou major projects of collaborative innovation in health care [201704020229], 2016 Guangdong provincial science and technology program on emergency preparedness of Zika [2016A020248001], Ministry of Science and Technology of China[2017ZX10305501-003] and the Municipal Science and Technology Bureau Foundation of Guangzhou [201508020106].

\section{Availability of data and materials}

The sequences used in this study are available in the GenBank (accession numbers for NS1: KY888678, KY911976, KY911978, KY911981, KY911982. accession numbers for E: KU820898, KJ438296, JX470186, KF824903, JQ822247). Raw data can be made available to interested researchers on request to the corresponding authors.

\section{Authors' contributions}

$X G, F Z$ and LY conceived the research project, designed the study protocol, interpreted data and wrote the manuscript. YW, CL and LZ performed experiments. JW, WH and CY managed patients, designed patients' follow-up and collected clinical samples required for this study. XJ critically reviewed and edited the manuscript. LY directed the entire research activities. All authors reviewed the results and approved the final version of the manuscript.

\section{Ethics approval and consent to participate}

The study was approved by the Ethical Committee of the Guangzhou Eighth People's Hospital (20131224). The samples were collected and used in strict accordance with Chinese rules and regulations for the protection of human subjects. All study subjects provided written informed consents for research use of their blood samples.

\section{Competing interests}

The authors declare that they have no competing interests.

\section{Publisher's Note}

Springer Nature remains neutral with regard to jurisdictional claims in published maps and institutional affiliations.

\section{Author details}

${ }^{1}$ Guangzhou Eighth People's Hospital, Guangzhou Medical University, 627 Dongfeng Rd. East, Guangzhou 510060, China. ${ }^{2}$ Viral Disease and Vaccine Translational Research Unit, CAS Key Lab of Molecular Virology and Immunology, Institut Pasteur of Shanghai, Chinese Academy of Sciences (CAS), Shanghai 200025, China.

Received: 25 January 2018 Accepted: 30 May 2018 Published online: 14 June 2018

References

1. Petersen LR, Jamieson DJ, Powers AM, Honein MA. Zika Virus. N Engl J Med. 2016;374:1552-63.

2. de Oliveira WK, Carmo EH, Henriques CM, Coelho G, Vazquez E, CortezEscalante J, et al. Zika virus infection and associated neurologic disorders in Brazil. N Engl J Med. 2017;376:1591-3.

3. Zhong YB, Liu XQ, Deng YC, Xu PH, Zhong GR, Zhang W. First case of laboratory-confirmed Zika virus infection imported into China. Chin Med J. 2016;129:2013-4. 
4. Liu L, Wu W, Zhao X, Xiong Y, Zhang S, Liu X, Qu J, Li J, Nei K, Liang M, et al. Complete genome sequence of Zika virus from the first imported case in mainland China. Genome Announc. 2016;4:e00291-16.

5. Zhang FC, Li XF, Deng YQ, Tong YG, Qin CF. Excretion of infectious Zika virus in urine. Lancet Infect Dis. 2016;16:641-2.

6. Yin Y, Xu Y, Su L, Zhu X, Chen M, Zhu W, et al. Epidemiologic investigation of a family cluster of imported ZIKV cases in Guangdong, China: probable human-to-human transmission. Emerg Microbes Infect. 2016;5:e100

7. Hong $W X$, Zhao $H$, Deng $Y Q$, Jiang $T$, $Y u X D$, Song $K Y$, et al. Severe dengue due to secondary DENV-1 infection in mainland China. J Clin Virol. 2013:57:184-6.

8. Zhao H, Zhao L, Jiang $T$, Li X, Fan H, Hong W, et al. Isolation and characterization of dengue virus serotype 2 from the large dengue outbreak in Guangdong, China in 2014. Sci China Life Sci. 2014;57:1149-55.

9. Zhao H, Zhang FC, Zhu Q, Wang J, Hong WX, Zhao LZ, et al. Epidemiological and Virological characterizations of the 2014 dengue outbreak in Guangzhou, China. PLoS One. 2016;11:e156548.

10. Roehrig JT. Antigenic structure of flavivirus proteins. Adv Virus Res. 2003;59: $141-75$.

11. Dejnirattisai W, Supasa P, Wongwiwat W, Rouvinski A, Barba-Spaeth G, Duangchinda T, et al. Dengue virus sero-cross-reactivity drives antibodydependent enhancement of infection with zika virus. Nat Immunol. 2016;17: $1102-8$

12. Stettler K, Beltramello M, Espinosa DA, Graham V, Cassotta A, Bianchi S, et al. Specificity, cross-reactivity, and function of antibodies elicited by Zika virus infection. Science. 2016;353:823-6.

13. Andrade DV, Harris E. Recent advances in understanding the adaptive immune response to Zika virus and the effect of previous flavivirus exposure. Virus Res. 2017; https://doi.org/10.1016/j.virusres.2017.06.019

14. Priyamvada L, Quicke KM, Hudson WH, Onlamoon N, Sewatanon J, Edupuganti $\mathrm{S}$, et al. Human antibody responses after dengue virus infection are highly cross-reactive to Zika virus. Proc Natl Acad Sci U S A. 2016:113:7852-7.

15. Yu L, Wang R, Gao F, Li M, Liu J, Wang J, et al. Delineating antibody recognition against Zika virus during natural infection. JCI Insight. 2017; 2:e93042.

16. Rastogi M, Sharma N, Singh SK. Flavivirus NS1: a multifaceted enigmatic viral protein. Virol J. 2016;13:131.

17. Hilgenfeld R. Zika virus NS1, a pathogenicity factor with many faces. EMBO J. 2016;35:2631-3.

18. Zhang B, Pinsky BA, Ananta JS, Zhao S, Arulkumar S, Wan $\mathrm{H}$, et al. Diagnosis of Zika virus infection on a nanotechnology platform. Nat Med. 2017;23:548-50

19. Balmaseda A, Stettler K, Medialdea-Carrera R, Collado D, Jin X, Zambrana JV, et al. Antibody-based assay discriminates Zika virus infection from other flaviviruses. Proc Natl Acad Sci U S A. 2017:114:8384-9.

20. Lustig Y, Zelena H, Venturi G, Van Esbroeck M, Rothe C, Perret C, et al. Sensitivity and kinetics of an NS1-based Zika virus enzyme-linked immunosorbent assay in Zika virus-infected travelers from Israel, the Czech Republic, Italy, Belgium, Germany, and Chile. J Clin Microbiol. 2017:55:1894-901.

21. Tsai WY, Youn HH, Brites C, Tsai JJ, Tyson J, Pedroso C, et al. Distinguishing secondary dengue virus infection from Zika virus infection with previous dengue by a combination of 3 simple serological tests. Clin Infect Dis. 2017;65:1829-36.

22. Muller DA, Young PR. The flavivirus NS1 protein: molecular and structural biology, immunology, role in pathogenesis and application as a diagnostic biomarker. Antivir Res. 2013:98:192-208.

23. Matheus S, Boukhari R, Labeau B, Ernault V, Bremand L, Kazanji M, et al. Specificity of dengue NS1 antigen in differential diagnosis of dengue and Zika virus infection. Emerg Infect Dis. 2016;22:1691-3.

24. Moore JP, Wallace LA, Follett EA, McKeating JA. An enzyme-linked immunosorbent assay for antibodies to the envelope glycoproteins of divergent strains of HIV-1. AIDS. 1989;3:155-63.

25. Pegu P, Vaccari M, Gordon S, Keele BF, Doster M, Guan Y, et al. Antibodies with high avidity to the gp120 envelope protein in protection from simian immunodeficiency virus SIV(mac251) acquisition in an immunization regimen that mimics the RV-144 Thai trial. J Virol. 2013;87:1708-19.

26. Tiller T, Meffre E, Yurasov S, Tsuiji M, Nussenzweig MC, Wardemann H. Efficient generation of monoclonal antibodies from single human B cells by single cell RT-PCR and expression vector cloning. J Immunol Methods. 2008:329:112-24.

27. Guan Y, Sajadi MM, Kamin-Lewis R, Fouts TR, Dimitrov A, Zhang Z, et al. Discordant memory B cell and circulating anti-Env antibody responses in HIV-1 infection. Proc Natl Acad Sci U S A. 2009;106:3952-7.
28. Wahala WM, Silva AM. The human antibody response to dengue virus infection. Viruses. 2011:3:2374-95.

29. Pasquier C, Joguet G, Mengelle C, Chapuy-Regaud S, Pavili L, Prisant N, et al. Kinetics of anti-ZIKV antibodies after Zika infection using two commercial enzyme-linked immunoassays. Diagn Microbiol Infect Dis. 2018;90:26-30.

30. Barzon L, Percivalle E, Pacenti M, Rovida F, Zavattoni M, Del Bravo P, et al. Virus and antibody dynamics in travelers with acute Zika virus infection. Clin Infect Dis. 2018;66:1173-80.

31. Chao DY, Galula JU, Shen WF, Davis BS, Chang GJ. Nonstructural protein 1-specific immunoglobulin $M$ and $G$ antibody capture enzyme-linked immunosorbent assays in diagnosis of flaviviral infections in humans. J Clin Microbiol. 2015;53:557-66.

32. Steinhagen K, Probst C, Radzimski C, Schmidt-Chanasit J, Emmerich P, van Esbroeck M, et al. Serodiagnosis of Zika virus (ZIKV) infections by a novel NS1-based ELISA devoid of cross-reactivity with dengue virus antibodies: a multicohort study of assay performance, 2015 to 2016. Euro Surveill. 2016; 21:e30426.

Ready to submit your research? Choose BMC and benefit from

- fast, convenient online submission

- thorough peer review by experienced researchers in your field

- rapid publication on acceptance

- support for research data, including large and complex data types

- gold Open Access which fosters wider collaboration and increased citations

- maximum visibility for your research: over $100 \mathrm{M}$ website views per year

At BMC, research is always in progress.

Learn more biomedcentral.com/submissions 